\title{
Potato starch resistivity as an inheritable trait
}

\author{
L.M. Gvozdeva ${ }^{1 *}$, I.V. Rozanova ${ }^{1,2}$, V.K. Khlestkin ${ }^{1,3}$, E.K. Khlestkina ${ }^{1,2}$ \\ 1 Institute of Cytology and Genetics, SB RAS, Novosibirsk, Russia \\ ${ }^{2}$ N.I. Vavilov All-Russian Research Institute of Plant Genetic Resources (VIR), St. Petersburg, Russia \\ ${ }^{3}$ Russian Research Institute of Farm Animal Genetics and Breeding - Branch of the L.K. Ernst Federal Science Center for Animal Husbandry, \\ St. Petersburg, Russia
}

DOI 10.18699/ICG-PlantGen2019-13

(c) Autors, 2019

*e-mail: ungersy@mail.ru

\begin{abstract}
Potato is a significant crop in many countries. It is not only a source of vitamins, amino acids and fiber, but also of starch. Starch is an important source of energetic food for humans, as well as an important raw material of organic origin for biochemical industry. In terms of enzyme digestibility, starch is subdivided into 3 fractions: rapidly digestible (DS), slowly digestible, and resistant starches (RS). RS passes through the stomach and small intestine, remaining intact, and then enters the large intestine, where it is fermented into useful short-chain fatty acids (SCFA: acetate, propionate and butyrate) and serves as an excellent nutrient medium for intestinal microflora. Unlike normal starch, RS increases the body's sensitivity to insulin and lowers blood glucose levels. Thus, a diet enriched for RS can be used both for weight maintenance / stabilization and for the treatment of obesity, diabetes, Crohn's and Bechterew's disease. Identification of genomic regions associated with potato starch resistivity indicates this property to be an inheritable trait. Data on the genomic regions will facilitate potato breeding for diverse purposes in human diet as well as in processing industry.
\end{abstract}

Key words: diabetes; digestible starch; genome-wide association studies; obesity; potato starch; resistant starch.

\section{Introduction}

Potato (Solanum tuberosum L.) is one of the most popular carbohydrate foods in industrialized and some developing countries, with its production in 2017 having reached 440 million tons (according to FAOSTAT). In addition to vitamins, amino acids, microelements, and fiber, potato is a source of starch. Starch consists of polysaccharides, molecules that are composed of a number of monosaccharides (glucose) motives linked together with $\alpha-1,4$ and/or $\alpha-1,6$ linkages. There are two main structural types of starch polysaccharides: amylose, which is a linear $\alpha-1,4$ molecule and typically constitutes $15-20 \%$ of starch, and amylopectin, which is a larger branched molecule with $\alpha-1,4$ and $\alpha-1,6$ linkages, a major component of starch.

Potato and potato products yield variable glycemic responses. For example, the published glycemic index (GI) values for potato vary from very low (23 for an unspecified cultivar) to very high (144 for boiled Desiree) (Foster-Powell, 2002). The variation in the GI values of potato-products may be due to a number of factors such as potato genotype, maturity, starch structure, processing, and storage conditions (Lynch, 2007).

In terms of digestibility, starch is typically divided into 3 fractions: rapidly digesting starch (RDS), slowly digesting starch (SDS), and resistant starch (RS). According to Englyst and others, RDS and SDS are the starch fractions that hydrolyze to dextrins by $\alpha$-amylase and pullulanase enzymes within 20 to 120 min after ingestion, respectively. RS is the portion that is not hydrolyzed after $120 \mathrm{~min}$ and passes from the small to the large intestine (Englyst, 1992), where it is fermented by gut microflora (Nugent, 2005). Chemically RS is measured as the difference between total starch (TS) and the sum of RDS and SDS:

$\mathrm{RS}=\mathrm{TS}-(\mathrm{RDS}+\mathrm{SDS})$

$\mathrm{RDS}+\mathrm{SDS}=\mathrm{DS}$ (digestible starch)
Thus, RS is considered excellent dietary fiber, which would be expected to confer benefits to gut health, particularly in the large intestine, where RS is fermented and can result in the release of gases (methane, hydrogen, carbon dioxide), shortchain fatty acids, or SCFAs, (formate, acetate, propionate, butyrate and valerate) and smaller amounts of organic acids (lactate and succinate) and alcohols (methanol and ethanol). The SCFA butyrate is of particular interest given its role as fuel for the cells lining the colon (colonocytes) and there is growing interest in the potential influence of gut bacteria on health outcomes, both locally in the gut and systemically (Kataoka, 2016).

It should be noted that the amount of RS in a given food varies for different individuals, as the exact time of the transition from the small to the large intestine fluctuates depending on an individual's gastrointestinal functioning, the concentrations of endogenous carbohydrate digestion enzymes, viscosity of the gastric fluid, as well as the food matrix itself.

There are several factors described to affect starch resistivity:

- amylose/amylopectin ratio; a high amylose content of starch is known to lower starch digestibility (Chung, 2009);

- length and branching of amylopectin chains; long linear chains of amylopectin provide starch resistivity, and the branched chains of amylopectin, starch digestibility (Benmoussa, 2007);

- retrogradation of amylose; retrograded amylose in wheat, maize, peas, and potatoes was found to be highly resistant to digestion (Ring, 1988);

- ions; potato starch gels showed a decreased yield of RS in the presence of ions like calcium and potassium, which may be reflected to the prevention of hydrogen bond formation between amylopectin and amylose chains; 
- interaction of starch polysaccharides with proteins; a mixture of potato starch and albumin was autoclaved and then cooled to $-20{ }^{\circ} \mathrm{C}$; it was found that added albumin reduced RS content (Escarpa, 1997);

- enzyme inhibitors; enzyme inhibitors like phytic acid, polyphenols, and lectins present in leguminous seeds have been found to inhibit in vitro digestion and, consequently, the glycemic index of starch; since phytic acid inhibits amylolysis, an increase in phytate content decreases starch digestibility (Thompson, 1984);

- size, smoothness and intactness of the granules; large, smooth, undamaged starch granules are more protected from effects of enzymes, since they are difficult to attach to the granule and get inside (Blazek, 2010).

So, starch resistivity is caused by a complex of factors, which together influence the digestibility of starch.

RS values in raw, not cooked potato are very high (70\% on average). Thus, raw potato RS causes the following beneficial effects on health:

1) RS increases SCFAs production. The SCFA butyrate is the preferred fuel for colonocytes, increasing colonic blood flow, lowering luminal $\mathrm{pH}$ and helping prevent the development of abnormal colonic cell populations as well as facilitating the maintenance of epithelial integrity and regulating inflammation (Kataoka, 2016).

2) Raw potato starch dramatically induces changes in microbial composition and microbial metabolites. Coprococcus, Ruminococcus, and Turicibacter (bacteria important for degradation of starch and SCFA production) increased significantly, while Sarcina and Clostridium decreased in relative abundances in the hindgut of pigs fed raw potato starch. Thus, raw potato starch not only alters the composition of the gut microbial community but also modulates the metabolic pathway of microbial metabolism (Sun, 2016).

3) RS consumption has been shown to improve glycemic control in both animal and human studies. Eating a RS-rich diet substantially increased the mobilization and use of fat stores as a consequence of reduction in insulin secretion. SCFAs are reported to inhibit adipose tissue lipolysis, and in the liver and muscles, particularly acetate was proposed to inhibit glycogenolysis, with the result of sparing carbohydrate and increasing fat oxidation. Since RS almost does not split into glucose in the small intestine and improves insulin sensitivity, it can be useful in such serious diseases as type 2 diabetes and obesity (Tapsell, 2004; Canfora, 2015).

4) Consumption of RS is beneficial to cardiovascular diseases. Particularly, it has been shown that RS is associated with cardiovascular risk reduction (Bernstein, 2013).

5) Considerable evidence exists suggesting a protective role of high-fibre diets in colon cancer. Fibre is suggested to reduce colon cancer risk by increasing fecal bulk, reducing transit time and diluting fecal contents (Murphy, 2012). High butyrate production is reported to reduce colon cancer risk and butyrate treatment of cultured colon cancer cells can blunt the proliferation of the cancer cells and stimulate apoptosis (Macfarlane, 2003).

A number of studies revealed that RS could have a positive role in intestinal absorption of minerals. In humans, an RS-rich diet substantially increased the apparent absorption of calcium and iron compared with a completely DS diet (Morais, 1996). This is likely one of the reasons why supplementation with resistant starch can protect bone density during weight loss and positively affect the immune system.

\section{Materials and methods}

Starch and DNA were isolated from 90 potato varieties and hybrids from the ICG "GenAgro" collection. Starch was tested for resistance to enzymatic digestion by $\alpha$-amylase. Genotyping data were obtained on a $22 \mathrm{~K}$ Illumina Potato SNP-array. The association mapping was performed with the use of software products TASSEL, Structure, R.

\section{Results and discussion}

Schonhals et al. (2016) showed that SNPs in the candidate genes Pain-1, InvCD141 (invertases), SSIV (starch synthase), StCDF1 (transcription factor), LapN (leucine aminopeptidase), and cytoplasm type are associated with potato tuber yield, starch content and/or starch yield.

Khlestkin et al. (2019) found several SNPs associated with starch amylopectin phosphorylation.

It has been found that key genes for starch biosynthesis may affect factors, resulting in variations in starch resistivity. Thus, granule-bound starch synthase gene (GBSS) knockout drastically increases amylopectin content to up to $>98 \%$. When inhibited, the SBEI gene encoding the main starch branching enzyme does not increase amylose content in modified potato. But simultaneous inhibition of both SBEI and SBEII genes results in high (60-89\%) amylose starch with minor amylopectin content. Elevation of SBEII expression allows obtaining starch characterized by increased amylopectin branching with shorter end chains. In contrast, amylopectin from potato plants with inhibited $S B E$ synthesis has longer polysaccharide chains with lower branching. GWD gene knockout results in amylopectin with reduced phosphate content and, accordingly, reduced viscosity gels from the modified starch. Low phosphate starch demonstrates also a reduced rate of biocatalytic hydrolysis. Overexpression of SSIV results in increased tuber starch content in both greenhouse and field grown plants. Starch granule morphology and crystallinity may be corrected at the genetic level as well (Khlestkin, 2017).

We performed a genome-wide association study to search for SNPs responsible for potato starch resistivity. Samples of starch from 90 potato varieties and hybrids from the ICG "GenAgro" collection were tested for resistance to enzymatic digestion by $\alpha$-amylase. The average starch resistivity value found was about $75 \%$ of the total amount of starch. Contrasting forms with low (40-52\%) and high (up to 99\%) resistance of starch to digestion were identified. The association mapping performed with the use of genotyping data obtained on the 22K Illumina Potato SNP-array revealed significant region on chromosome 5 .

\section{Conclusions}

In conclusion, the potato tuber starch set studied contains starch samples with both high and low RS content, which makes it possible to differentiate potato varieties according to this trait. Resistance towards amylases is a very important trait related to starch as a tunable nutrient, probably connected to 
several human diseases, and thus has to be carefully taken into account in diets for treatment and prophylactics of the diseases and comorbid states. Potato varieties with starch with the trait 'contrasting resistance' were identified. The SNP found on chromosome 5 is associated with the trait 'starch digestibility' and reflects the participation of this chromosome in the formation of resistance of potato starch towards amylase digestion. Further research is being conducted on the identified genomic region, aimed at identifying a candidate gene and developing a convenient PCR marker for accelerated breeding of potatoes with different digestibility of starch.

\section{References}

Benmoussa M., Moldenhauer K., Hamaker B. Rice Amylopectin Fine Structure Variability Affects Starch Digestion Properties. J. Agric. Food Chem. 2007;55:1475-1479.

Bernstein A.M., Titgemeier B., Kirkpatrick K., Golubic M., Roizen M.F. Major cereal grain fibers and psyllium in relation to cardiovascular health. Nutrients. 2013;5:1471-1487.

Blazek J., Gilbert E.P. Effect of enzymatic hydrolysis on native starch granule structure. Biomacromolecules. 2010;11:3275-3289.

Canfora E., Jocken J., Blaak E. Short-chain fatty acids in control of body weight and insulin sensitivity. Nat. Rev. Endocrinol. 2015; 11:577-591.

Chung H., Liu Q., Hoover R. Impact of annealing and heat-moisture treatment on rapidly digestible, slowly digestible and resistant starch levels in native and gelatinized corn, pea and lentil starches. Carbohydr. Polym. 2009;75:436-447.

Englyst H, Kingman S.M., Cummings J.H. Classification and measurement of nutritionally important starch fractions. Eur. J. Clin. Nutr. 1992;46(S2):33-50.

Escarpa A., Gonzalez M.C., Morales M.D., Saura-Calixto F. An approach to the influence of nutrients and other food constituents on resistant starch formation. Food Chem. 1997;60:527-536.

Foster-Powell K., Holt S.H.A., Brand-Miller J.C. International tables of glycemic index and glycemic load values. American Journal Clinical Nutrition. 2002;76:5-56.

Kataoka $\mathrm{K}$. The intestinal microbiota and its role in human health and disease. Journal Medical Investigation. 2016;63:27-37.

Khlestkin V.K., Peltek S.E., Kolchanov N.A. Target genes for development of potato (Solanum tuberosum L.) cultivars with desired starch properties (review). Sel'skokhozyaistvennaya Biologiya $=$ Agricultural Biology. 2017;52(1):25-36.
Khlestkin V.K., Rozanova I.V., Efimov V.M., Khlestkina E.K. Starch phosphorylation associated SNPs found by genome-wide association studies in the potato (Solanum tuberosum L.). BMC Genet. 2019;18:20-29.

Lynch D., Liu Q., Tarn T. R., Bizimungu B., Chen Q., Harris P. et al. Glycemic index - a review and implications for the potato industry. American Journal of Potato Research. 2007;84(2):179-190.

Macfarlane S., Macfarlane G.T. Regulation of short-chain fatty acid production. Proceedings Nutrition Society. 2003;62:67-72.

Morais M.B., Feste A., Miller R.G, Lifichitz C.H. Effect of resistant starch and digestible starch on intestinal absorption of calcium, iron and zinc in infant pigs. Paediatr. Res. 1996;39:872-876.

Mosquera T., Alvarez M.F., Jimenez-Gomez J.M., Muktar M.S., Paulo M.J., Steinemann S. et al. Targeted and untargeted approaches unravel novel candidate genes and diagnostic SNPs for quantitative resistance of the potato (Solanum tuberosum L.) to Phytophthora infestans causing the late blight disease. PLoS One. 2016; 11(6): 0156254.

Murphy N., Norat T., Ferrari P., Jenab M., Bueno-de-Mesquita B., Skeie G. et al. Dietary fibre intake and risks of cancers of the colon and rectum in the European prospective investigation into cancer and nutrition (EPIC). PLoS One. 2012;7:e39361.

Nugent A.P. Health properties of resistant starch. Nutr. Bull. 2005;30: $27-54$.

Ring S.G., Gee J.M., Whittam M., Orford P., Johnson I. Resistant Starch. Its chemical form in foodstuffs and effect on digestibility in vitro. Food Chem. 1998;28:97-109.

Schonhals E.M., Ortega F., Barandalla L., Aragones A., Ruiz de Galarreta J.I., Liao J.C. et al. Identification and reproducibility of diagnostic DNA markers for tuber starch and yield optimization in a novel association mapping population of potato (Solanum tuberosum L.). Theoretical Applied Genetics. 2016;129(4):767-785.

Sun Y., Su Y., Zhu W. Microbiome-metabolome responses in the cecum and colon of pig to a high resistant starch diet. Front Microbiol. 2016;7:779.

Tapsell L.C. Diet and metabolic syndrome: Where does resistant starch fit in? J. Association Analyt. Chem. Int. 2004;87:756-760.

Thompson L.U., Yoon J.H. Starch digestibility as affected by polyphenol and phytic acid. J Food Sci. 1984;49:1268-1269.

Acknowledgements. The work supported by a grant from the RFBR (No. 17-29-08006).

Conflict of interest. The authors declare no conflict of interest. 